\title{
Endovascular targeted coil embolization using dual vessel approach for traumatic direct carotid- cavernous fistula following severe head trauma: technical notes
}

\author{
JUNYA TSURUKIRI ${ }^{1}$, TOSHIAKI ONITSUKA ${ }^{2}$, KEN MATSUSHIMA $^{2}$, EITARO OKUMURA $^{1}$, HIROYUKI JIMBO $^{1}$
}

1 Emergency and Critical Care Medicine, Tokyo Medical University Hachioji Medical Center, Tokyo, Japan

2 Neurosurgery, Tokyo Medical University Hachioji Medical Center, Tokyo, Japan

Corresponding author:

Junya Tsurukiri

Emergency and Critical Care Medicine

Tokyo Medical University Hachioji Medical Center

1163 Tatemachi, Hachioji. Tokyo 193-0998. Japan

Phone: $+81-426655611$

Fax: $+81-426655687$

E-mail: junya99@tokyo-med.ac.jp

\section{ABSTRACT}

Direct carotid cavernous fistulas (dCCFs) represent different entitles from spontaneous cavernous sinus (CS) dural arteriovenous fistulas (CSAVFs). Traumatic dCCFs are direct arteriovenous communications between the internal carotid artery (ICA) and cavernous sinus, resulting from a traumatic tear of the horizontal or posterior ascending intracavernous segment of the ICA by skull fracture fragments. They are account for $0.2-0.3 \%$ of craniofacial trauma and a serious problem that results in neuro-ophthalmologic symptoms. Intracerebral hemorrhage followed by cortical venous reflux is a most serious complication. Although embolization of dCCFs with detachable balloons was previously accepted worldwide as the firstline therapeutic option because it is technically simple, these devices are currently unavailable and other treatment options have included coil embolization of the fistula to preserve the internal carotid artery. Therefore, sinus packing by coil embolization is now a first-line therapy, but there is the disadvantage of neuro-ophthalmologic symptoms by nerve palsy occurred in CS. In this study, we treated two patients with dCCFs caused by severe head trauma who underwent dual vessel approach to endovascular targeted coil embolization and successful resolution. We concluded that dual vessel approach to endovascular targeted coil embolization is an effective and safety technique among patients with traumatic dCCFs.
Key words: endovascular treatment, brain injury, dural arteriovenous fistulas, cavernous sinus, superior ophthalmic vein

\section{INTRODUCTION}

Traumatic direct carotid-cavernous fistulas (dCCFs) have direct arteriovenous communications between the internal carotid artery (ICA) and cavernous sinus (CS), resulting from a traumatic tear of the ICA by skull fracture fragments arising from craniofacial trauma. (1) Clinical signs and symptoms such as cortical venous reflux or refractory ocular symptoms, which are associated with poor outcomes, warrant emergency intervention. Technical advancements in endovascular equipment and embolization agents have contributed to the acceptance of endovascular treatment of dCCFs as an effective and safe method. Although dense packing of the entire CS by coil embolization is currently regarded as a first-line therapy to close the fistula, tighter and more dense packing of smaller areas of the CS using targeted coil embolization is a reasonable treatment strategy. (2-4)

\section{CASE PRESENTATIONS}

Case 1. A 45-year-old man was admitted to our emergency department after a motor vehicle accident. His previous medical history was unremarkable. On examination, he had a Glasgow Coma Scale (GCS) score of eye E1, verbal V1, motor M4, and the di- ameter of his pupils was $3 \mathrm{~mm}$. Computed tomography (CT) revealed cranio-facial fractures, traumatic subarachnoid hemorrhage, and severe lung injuries. The patient underwent intracranial pressure (ICP) monitoring, the initial ICP was $21 \mathrm{cmH} 2 \mathrm{O}$. His ICP was consistently maintained at below $20 \mathrm{cmH} 2 \mathrm{O}$ in the intensive care unit. On day 14 after injury, the patient underwent maxillomandibular fixation for facial fractures. On day 16, his physical examination revealed chemosis, conjunctival hyperemia, and exophthalmos of the left eye. Contrast-enhanced CT (CECT) images revealed enhancement of the left CS and left superior ophthalmic vein (SOV) (figure $1 \mathrm{~A}, \mathrm{~B})$. Diagnostic angiography demonstrated drainage of the dCCF directly into the left CS, SOV, intercavernous sinus, and bilateral inferior petrosal sinus (IPS) (figure 1C,D). A fistula at the cavernous portion of the left ICA was detected using three-dimensional (3D) digital subtraction angiography (figure 1C). A balloon occlusion test (BOT) was performed to confirm the tolerance of the patient to temporary interruption of blood flow; however, after 15 minutes of the test, neurological examination was abnormal.

Endovascular treatment was performed under general anesthesia. The patient underwent systemic heparinization, and activated clotting time (ACT) was maintained between 200 and 300 s. First, a trans-arterial approach was adopted with a 7-Fr. guiding catheter (Launcher, Medtronic, Tokyo, JP) placed into the left ICA via a right femoral approach, followed by superselective catheterization into the CS from the ICA 
through the fistula using a microcatheter (Headway, Termo, Tokyo, JP) (figure 2A). Subsequently, a trans-venous approach was adopted using a 6-Fr. guiding catheter (Launcher, Medtronic, Tokyo, JP) inserted into the internal jugular vein via a right femoral approach, followed by superselective catheterization of the left CS around the fistula through the left IPS using another microcatheter (SL-10, Stryker, Tokyo, JP) (figure 2B). Coil embolization was initiated via a trans-arterial microcatheter using 3D microcoils deployed in the left $\mathrm{CS}$ around the fistula (figure 2C). Continuous packing into the initial coil proceeded smoothly in a distal-to-proximal approach until the microcatheter was deviated to the ICA (figure 2D). After trans-arterial embolization, coil embolization was additionally performed to fill the coil mass tighter using the trans-venous microcatheter in a distal-to-proximal approach until the fistula flow completely ceased. We demonstrated this by performing angiography of the right ICA, showing that targeted coil embolization had been achieved (figure 2E). Although a guidewire balloon catheter was introduced into the ICA to deal with accidental bleeding and as a marker of fistulous location, we did not inflate it. The patient's symptoms resolved rapidly in the following 5 days and he was discharged without further complications. He continues to remains free of symptoms 2 years after embolization.

Case 2. A 47-year-old man was transferred to our neurosurgery department from another hospital. He had suffered craniofacial fractures, traumatic subarachnoid hemorrhage, and a work-related brain injury 5 months earlier. On examination, he had a GCS score of E2V1M4. His physical examination revealed chemosis, conjunctival hyperemia, and exophthalmos of the right eye. CECT images revealed a marked enhancement of the CS and right SOV (figure 1D-F). Diagnostic angiography demonstrated dCCF draining directly into the right $\mathrm{CS}, \mathrm{SOV}$, intercavernous sinus, inferior ophthalmic vein, and superficial cerebral vein. (figure 4C,D). A fistula was detected at the clinoid portion of the right ICA (figure 3A). BOT was positive, resulting in an abnormal neurological examination.

Endovascular treatment was performed under general anesthesia. The patient underwent systemic heparinization with ACT of 200-300 s. First, a trans-venous approach, via direct puncture of the right SOV, was performed, followed by superselective catheterization into the CS around the fistula using a microcatheter (Echelon,

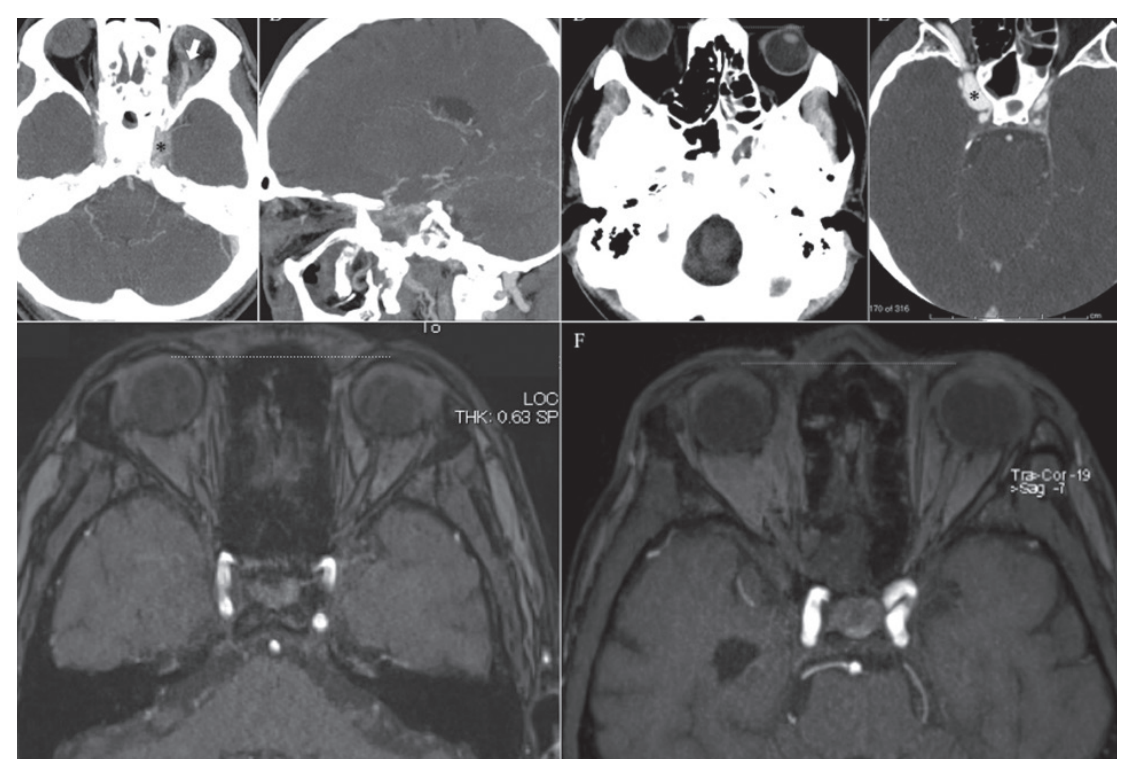

Figure 1.

Case 1. Contrast-enhanced CT imaging reveals an enhancement of the cavernous sinus (black ${ }^{*}$ ), and right superior ophthalmic vein (white arrow) (A), and the fistula (black arrow) as an indication of a carotid-cavernous fistula (B). Magnetic resonance imaging (MRI) revealed no revascularization after 2 years from the onset (C).

Case 2. Contrast-enhanced CT imaging reveals a proptosis and an enhancement of the cavernous sinus (black ${ }^{\star}$ ), and right superior ophthalmic vein (white arrow) (D, E).

Magnetic resonance imaging (MRI) revealed no revascularization after 1 year from the onset $(\mathrm{F})$.

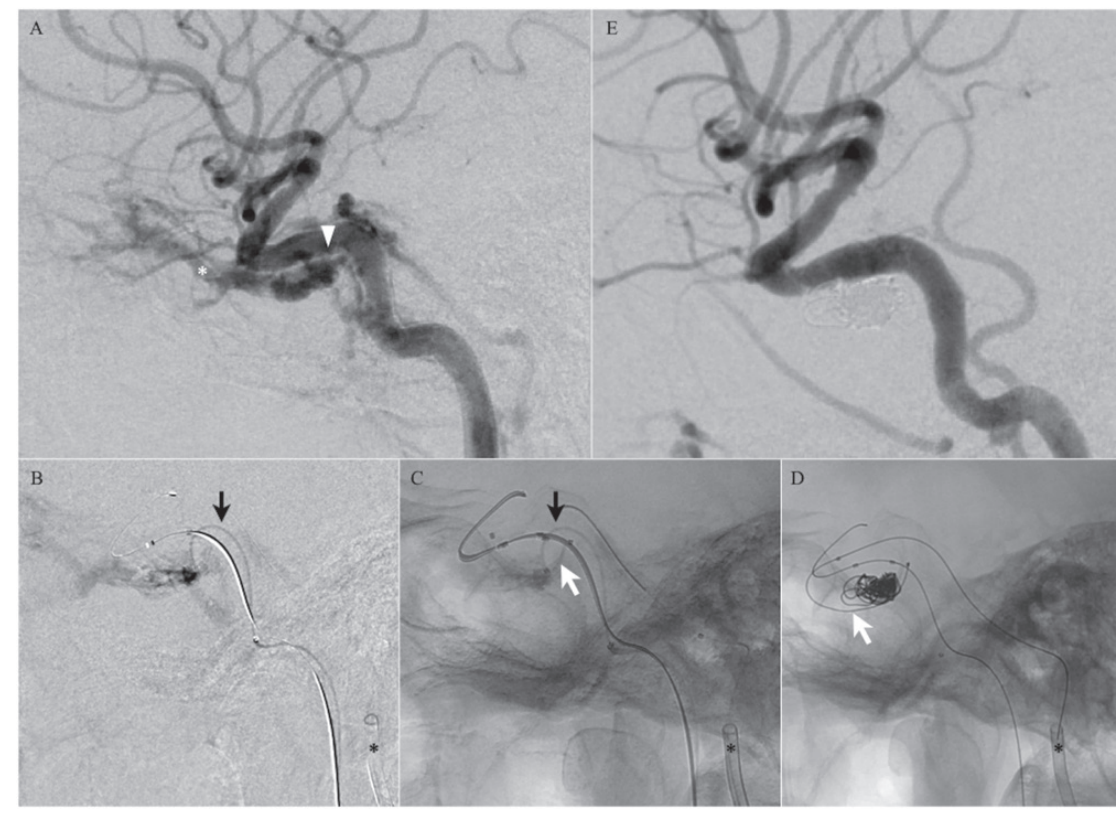

Figure 2.

Left carotid artery angiogram showing a left carotid-cavernous fistula with multiple routes of venous drainage (A). A fistula detected at the clinoid portion of the right internal carotid artery (white arrow head), and the superior ophthalmic vein (white ${ }^{\star}$ ). A trans-arterial microcatheter into the CS through the fistula (B, C; black arrow) and a trans-venous microcathter into the cavernous sinus (CS) (C, D; white arrow) through a guiding catheter $\left(^{*}\right)$. Packing of partial compartment around the fistula of the CS using trans-arterial microcatheter (D). Left internal carotid artery (ICA) angiogram revealed that the fistula flow completely ceased with reduction of the number of coils used (E). 


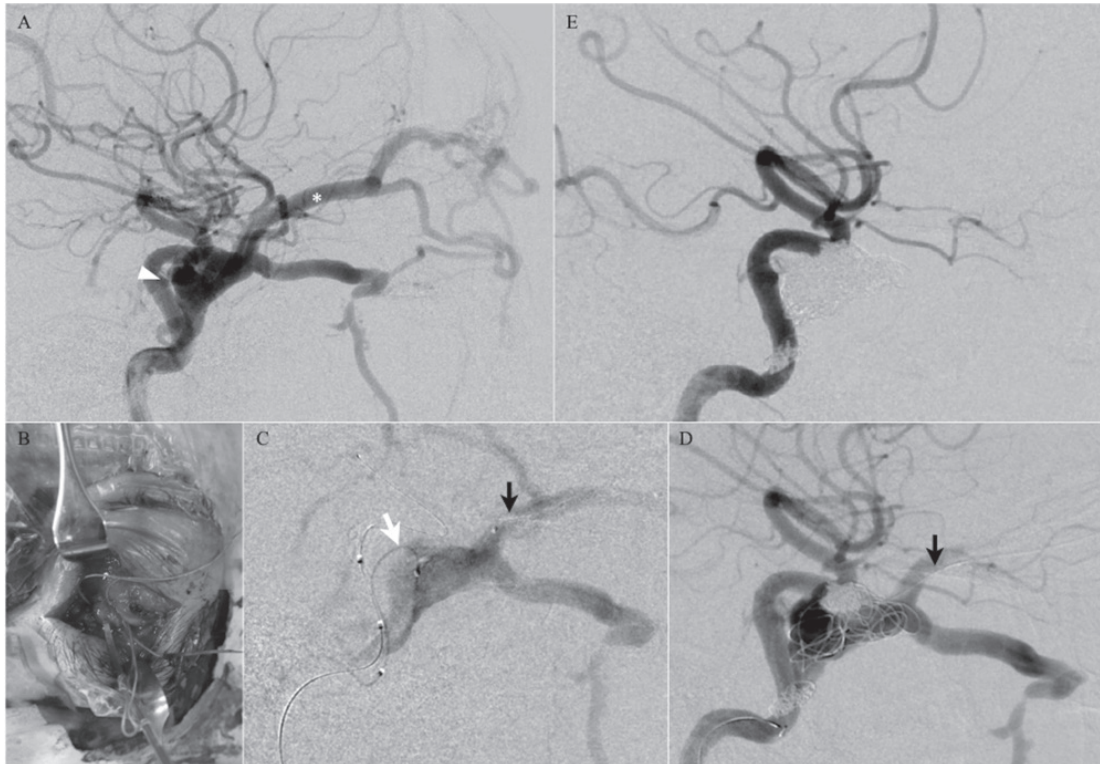

Figure 3.

Right carotid artery angiogram showing a right carotid-cavernous fistula with multiple routes of venous drainage (A). A fistula detected at the clinoid portion of the right internal carotid artery (white arrow), and the superior ophthalmic vein (white ${ }^{\star}$ ). Photograph showing direct puncture of the right superior ophthalmic vein (arrow) using an 18-gauge puncture needle (B). A trans-orbital microcathter into the cavernous sinus (CS) (C, D; black arrow), and a trans-arterial microcatheter into the CS through the fistula (c; white arrow). An uncal vein outflow occlusion (d; arrow head), and packing of partial compartment of the CS (D). Right ICA angiogram revealed that the fistula flow completely ceased with reduction of the number of coils used (E).

Covidien, Tokyo, JP) (figure 3B). Subsequently, a trans-arterial approach was performed, with a 6-Fr. guiding catheter (Launcher, Medtronic, Tokyo, JP) placed into the right ICA via a right femoral approach, followed by superselective catheterization from the ICA into the CS through the fistula using a microcatheter (Headway, Termo, Tokyo, JP) (figure 3C). First, coil embolization was performed via trans-orbital microcatheter using $3 \mathrm{D}$ microcoils deployed in the left CS around the fistula. Continuous packing of the CS proceeded smoothly in a distal-to-proximal approach (figure 3D). After trans-venous embolization, coil embolization was densely packed using the trans-arterial microcatheter in a distal-to-proximal approach until the fistula flow ceased. This was demonstrated by angiography of the right ICA, showing that targeted coil embolization had been achieved (figure 3E). The patient's symptoms resolved rapidly in the following 4 days, and he was discharged to the previous hospital without further complications. He remains free of symptoms 1 year after embolization.

\section{DISCUSSION}

To the best of our knowledge, this is the first report accompanied by radiological images of a dual vessel approach to endovascular targeted coil embolization for a traumatic dCCF following severe multiple traumas. Traumatic dCCF is different from spontaneous CS dural arteriovenous fistulas (CSAVFs), and it can result in significant neuro-ophthalmologic morbidities, such as motor paralysis, unconsciousness, and blindness as the disease progresses, or mortality. $(1,2)$ Spontaneous CSAVFs are often complicated, with a bilateral arterial or both internal and external carotid artery supply. The feeding artery is often so tiny that the trans-arterial approach remains a technical challenge. dCCFs have direct arteriovenous communications between the ICA and the CS, resulting from a traumatic tear of the ICA by skull fracture fragments arising from craniofacial trauma. dCCFs account for $0.2 \%-0.3 \%$ of craniofacial trauma, (1) and intracerebral hemorrhage is the most serious complication. As a result of a traumatic tear of the horizontal or posterior ascending intracavernous segment of the ICA, CS and SOV dilation are signs indicative of arteriovenous shunt in dCCFs; additionally, CSAVFs generally present with a low flow compared with the high flow seen in dCCF.

Endovascular treatment of dCCFs is generally accepted as a safe and effective method owing to technological advancements in endovascular equipment and embolization agents. (2-4) Although embolization of CS with technically simple, detachable balloons was previously accepted worldwide as the best therapeutic option, these devices are presently unavailable; currently, other treatment options include coil embolization of the fistula to preserve the ICA. $(5,6)$ Dense packing of the whole CS by coil embolization is now considered to be first-line therapy, but cranial nerve palsy sometimes occurs as a result. Analysis of the long-term outcome of patients undergoing coil embolization of CSAVFs revealed that up to $44 \%$ of patients had persistent cranial nerve deficits with oculomotor disturbance and visual dysfunction. (7) These permanent symptoms were associated with the space-occupying effect of the coil density in CS, and a coil volume of $>200 \mathrm{~mm} 3$ was reported to be a critical factor. $(7,8)$ Furthermore, dense packing of large areas of the CS includes not only the element of high cost for the amount of coils used but also difficulties in determining the optimal volume of coils. Therefore, as an alternative to dense CS packing, targeted coil embolization aimed at treating only the affected area is a reasonable strategy to reduce the number of coils used; in addition, morbidity rates are decreased by decreasing coil volume. (4) For targeted coil embolization, a careful delineation of the precise fistulous point is essential. Although dCCF are difficult to diagnose, the patient's clinical history and magnetic resonance imaging (MRI) results are very important tools for diagnosis. MRI is more sensitive than CT imaging in the detection of radiological signs in patients with traumatic brain injury. However, a recent report demonstrated that the detection of SOV dilation on CT occurs in $86 \%-100 \%$ of cases, which is similar to detection rates of MRI T2-weighted sequences (75\%$100 \%)$. (9) In the present study, we detected the fistulous point using CECT. Therefore, when dCCFs are suspected in patients with head trauma, obtaining multiplanar reconstruction from CECT images is useful to ascertain the complex arterial supply and optimize decisions for therapy. (10)

Methods of coil embolization using a double microcatheter technique were first reported by Baxter et al. for the treatment of cerebral aneurysm, particularly large or wide-necked aneurysms. $(11,12)$ If there 
is any evidence of coil instability within the aneurysm or significant impingement of coil loops on the parent artery was observed in the initial attempts at embolization, this technique enables interventionalists to advance a second microcatheter into the aneurysm, allowing two coils to be braced across the aneurysmal neck before the detachment of either coil. The main goal of endovascular treatment is to occlude the tear (fistula) between the ICA and CS and preserve the patency of the ICA. Coil embolization using a balloonassisted technique is generally accepted in $\mathrm{dCCFs}$ to prevent coil protrusion or migration into the ICA. However, it may be difficult to perform this technique in patients with no cerebral tolerance for circulating arrests of the ipsilateral ICA. $(13,14)$ In this study, both patients had positive BOT results; therefore, balloon-assisted coil embolization was unsuitable due to the high risk of complications. Therefore, we performed a double microcatheter/dual vessel technique for targeted coil embolization to preserve the patency of the ICA, and the results were successful without using a balloon.

Although covered stents, liquid embolic agents, and microvascular plugs may be an alternative treatment option for dCCFs without a space-occupying effect, the available stents are inflexible and have not been approved for this indication. (15)

\section{CONCLUSION}

Dual vessel approach to endovascular targeted coil embolization appears to be safe, and targeted coil embolization technique is effective among patients with traumatic dCCF caused by severe head trauma an as an adjunct for sinus packing.

\section{ACKNOWLEDGMENTS}

The authors would like to thank Enago (www.enago.jp) for the English language review.

\section{REFERENCES}

1. Higashida RT, Halbach VV, Tsai FY, Norman D, Pribram HF, Mehringer CM, et al. Interventional neurovascular treatment of traumatic carotid and vertebral artery lesion: results in 234 cases. AJR Am J Roentgenol 1989 Sep;153(3):577-82. DOI: 10.2214/ ajr.153.3.577.

2. Korkmazer B, Kocak B, Tureci E, Islak C, Kocer N, Kizilkilic O. Endovascular treatment of carotid cavernous sinus fistula: a systematic review. World J Radiol 2013 Apr;5(4):143-55. doi: 10.4329/wjr.v5.i4.143.

3. Lu X, Hussain M, Ni L, Huang Q, Zhou F, Gu Z, et al. A Comparison of different transarterial embolization techniques for direct carotid cavernous fistulas: a single center experience in 32 patients. J Vasc Interv Neurol 2014 Dec;7(5):35-47.

4. Agid R, Willinsly RA, Haw C, Souza MP, Vanek IJ, terBrugge KG. Targeted compartmental embolization of cavernous sinus dural arteriovenous fistulae using transfemoral medial and lateral facial vein approaches. Neuroradiology 2004 Feb;46(2):156-60.

5. Wang W, Li YD, Li MH, Tan HQ, Gu BX, Wang J, et al. Endovascular treatment of post-traumatic direct carotid-cavernous fistulas: A single-center experience. J Clin Neurosci 2011 Jan;18(1):24-8. doi: 10.1016/j.jocn.2010.06.008.

6. Niu Y, Li L, Tang J, Zhu G, Chen Z. Embolization of direct carotid cavernous fistulas with the novel double-balloon technique. Interv Neurodaiol 2016 Apr;22(2):201-5. doi: 10.1177/1591019915609779.

7. Bink A, Goller K, Lüchtenberg M, Neumann-Haefelin, Dützmann S, Zanella F, et al. Long-term outcome after coil embolization of cavernous sinus arteriovenous fistulas. AJNR Am J Neuroradiol 2010 Aug;31(7):1216-21. doi: 10.3174/ajnr.A2040

8. Nishino K, Ito Y, Hasegawa H, Kikuchi B, Shimbo J, Kitazawa K, et al. Cranial nerve palsy following transvenous embolization for a cavernous sinus dural arteriovenous fistula: association with the volume and location of detachable coils. J Neurosurg 2008 Aug;109(2):208-14. doi: 10.3171/JNS/2008/109/8/0208.

9. Dos Santos D, Monsignore LM, Nakiri GS, Cruz AA, Colli BO, Abud DG. Imaging diagnosis of dural and direct cavernous carotid fistulae. Radiol Bras 2014 Jul-Aug;47(4):251-5. doi: 10.1590/0100-3984.2013.1799.

10. Lee JY, Jung C, Ihn YK, Kim DJ, Seong SO, Kwon BJ. Multiditector CT angiography in the diagnosis and classification of carotidcavernous fistula. Clin Radiol 2016 Jan;71(1):e64-71. doi: 10.1016/j.crad.2015.10.018

11. Baxter BW, Rosso D, Lowine SP. Double microcatheter technique for detachable coil treatment of large, wide-necked intracranial aneurysms. Am J Neuroradiol 1998 Jun-Jul;19(6):1176-8.

12. Shin YS, Kim D, Lee SI, Chung JI, Yoon PH, Lee KC. The Usefulness of the New "Double-Catheter Technique" in the Treatment of Parent Artery Incorporated Wide-Necked Aneurysm with Guglielmi Detachable Coils. Interv Neuroradiol 2000 Mar;6(1):61-4.

13. Andrade G, Ponte De Souza ML, Marques R, Silva JL, Abath C, Azevedo-Filho HR. Endovascular treatment of traumatic carotid cavernous fistula with balloon-assisted sinus coiling. A technical description and initial results. Interv Neuroradiol 2013 Dec;19(4):44554 .

14. De Renzis A, Nappini S, Consoli A, Renieri L, Limbucci N, Rosi A, et al. Balloon-assisted coiling of the cavernous sinus to treat direct carotid cavernous fistula. A single center experience of 13 consecutive patients. Interv Neuroradiol 2013 Sep;19(3):344-52.

15. Nossek E, Zumofen D, Nelson E, Raz E, Potts MB, Desousa KG, et al. Use of pipeline embolization devices for treatment of direct carotid-cavernous fistula. Acta Neurochir 2015 Jul;157(7):1125-9. doi: 10.1007/s00701-015-2446-0. 